\title{
What do we know about the HIV risk characteristics of adolescent girls and young women in Zambia? Findings from DREAMS implementation science research
}

Population Council

Follow this and additional works at: https://knowledgecommons.popcouncil.org/departments_sbsr-hiv

Part of the Demography, Population, and Ecology Commons, Family, Life Course, and Society Commons, International Public Health Commons, and the Women's Health Commons How does access to this work benefit you? Let us know!

\section{Recommended Citation}

Population Council. 2018. "What do we know about the HIV risk characteristics of adolescent girls and young women in Zambia? Findings from DREAMS implementation science research," DREAMS Results Brief. Washington, DC: Population Council. 


\section{WHAT DO WE KNOW ABOUT THE HIV RISK CHARACTERISTICS OF ADOLESCENT GIRLS AND YOUNG WOMEN IN ZAMBIA?}

FINDINGS FROM DREAMS IMPLEMENTATION SCIENCE RESEARCH

AIDS is the leading cause of death among adolescent girls and young women (AGYW) in Sub-Saharan Africa. The Population Council is conducting implementation science research in Zambia and other countries across sub-Saharan Africa to build needed evidence to inform community-based, girl-centered HIV prevention programming. Critical to this process is understanding AGYW's knowledge, attitudes, sexual behaviors, and characteristics of their sexual partnerships that may put them at risk of HIV acquisition.

This results brief summarizes key findings from a crosssectional survey of 1,915 AGYW, half of whom were 15-19 years old and the other half 20-24 years old, residing in urban districts of Lusaka and Ndola. The survey was conducted between November 2016 and April 2017 in locations where the DREAMS program is being implemented. The findings in this brief were shared at a data interpretation workshop in Lusaka in June 2017. Subsequent data collection with AGYW DREAMS program participants will assess the influence of the program in reducing their HIV vulnerability.

Location: Kalingalinga in Lusaka and Lubuto in the Ndola district (within Copperbelt province)

Study Duration: $2016-2018$

Funder: Bill \& Melinda Gates Foundation

For more information, contact Sanyukta Mathur (smathur@popcouncil.org) or Maurice Musheke (mmusheke@popcouncil.org).

\section{KEY FINDINGS}

Half or more AGYW lack comprehensive knowledge of HIV and AIDS.

Nearly a third of adolescent girls and threefourths of young women report having had sex; and among sexually active AGYW, their condom use at last sex was low.

Although most young women had recently tested for HIV and know their HIV status, fewer adolescent girls had recently tested for HIV. Among AGYW who report being HIV positive, most were on treatment.

Many AGYW report experiencing sexually transmitted infection (STI) symptoms, but a sizable proportion of AGYW have not sought treatment.

Male partners are on average five years older than their AGYW partner, have been with their AGYW partners for more than a year, and onefourth of them engaged in jobs associated with high HIV risk (e.g., miner, trucker).

Nearly one-fourth of AGYW had experienced either physical or sexual violence from their partner in the last year and a majority of AGYW anticipated abuse from their male partners if they tested HIV positive and disclosed to their partner.

Two-thirds of young women and half of the adolescent girls knew their partner's HIV status. 


\section{RESULTS}

Who are the survey respondents?
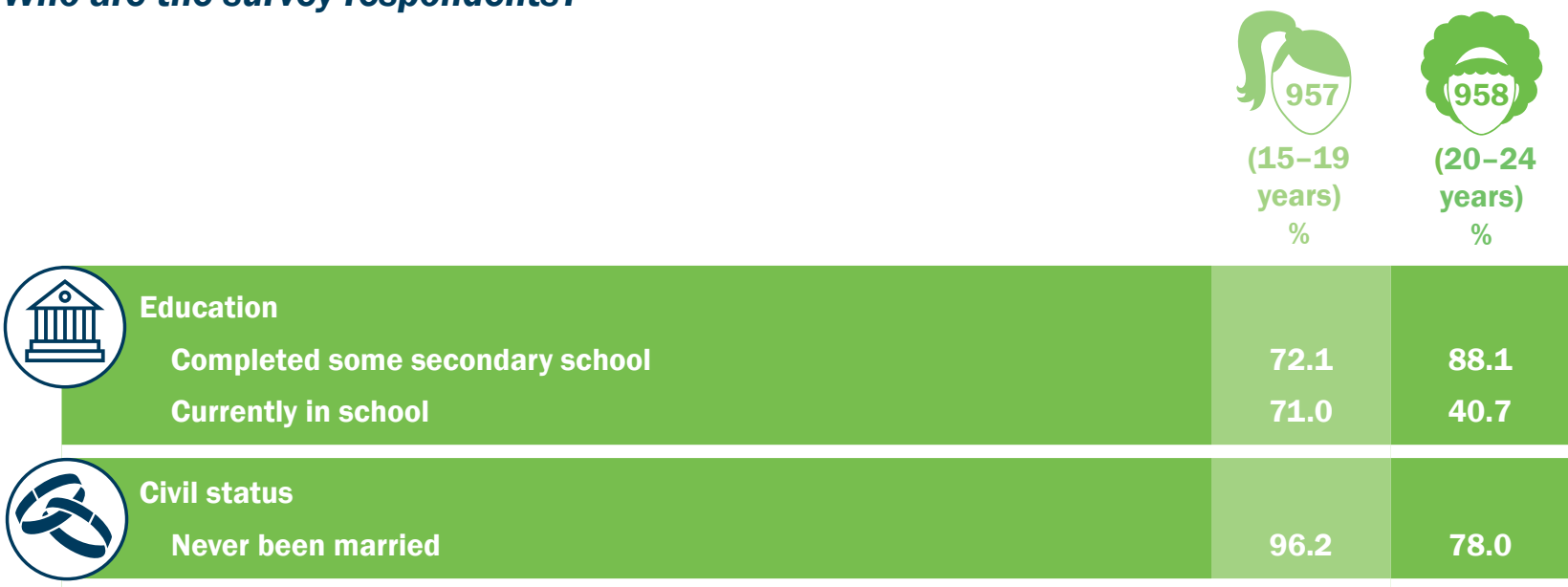

Civil status

Never been married

96.2

78.0

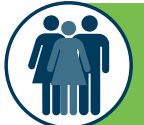

Parental loss

At least one parent

36.8

48.5

Both parents

7.6

13.3

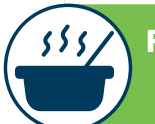

Food security

Gone without eating at least a whole day and night in the past month

19.5

17.4

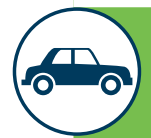

Mobility

Lived in their community for 5 or more years

61.8

51.3

Travel outside their community at least once a month

23.3

35.4

\section{What do AGYW know about HIV and AIDS?}

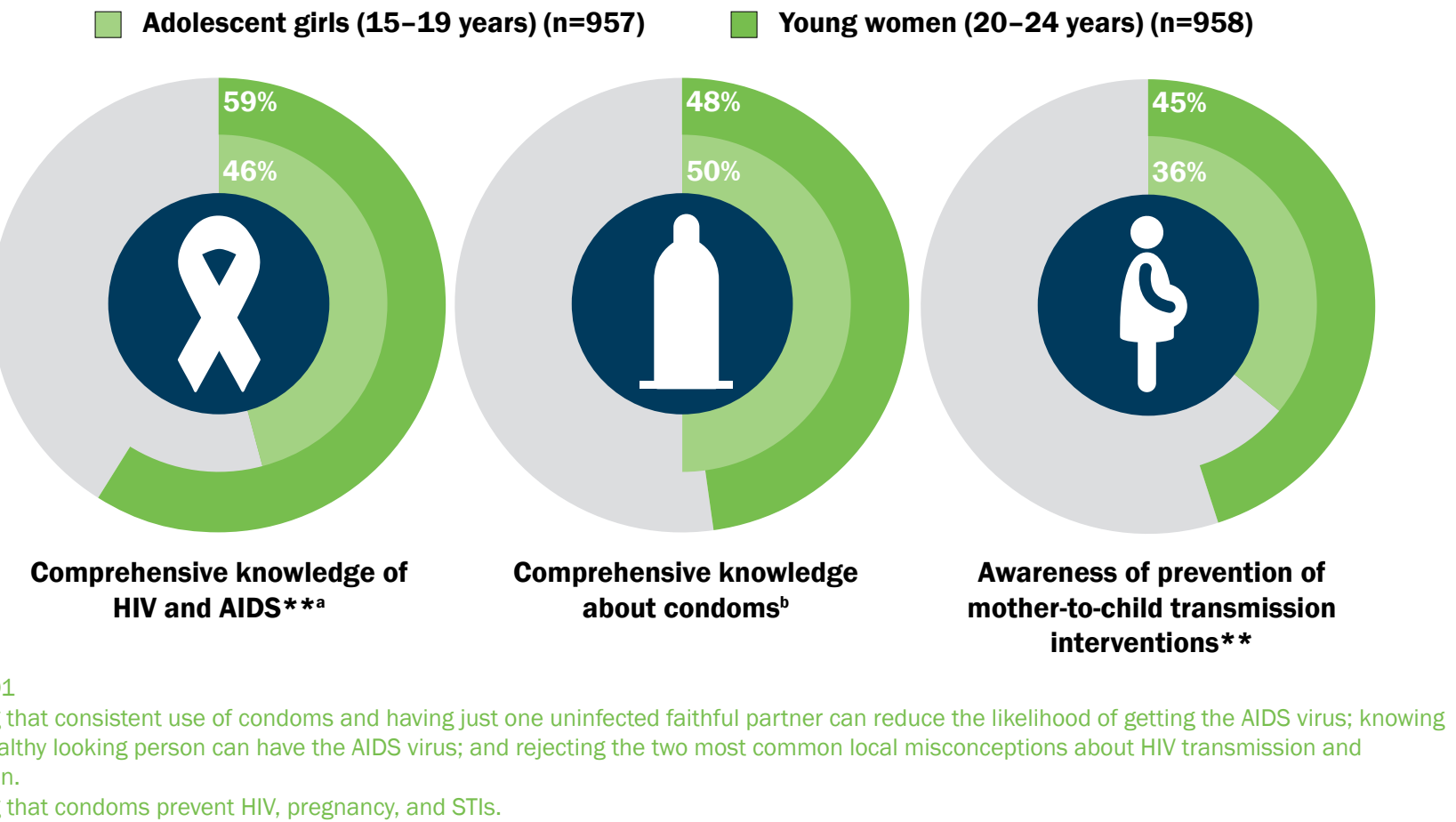


What are the sexual behaviors of AGYW?

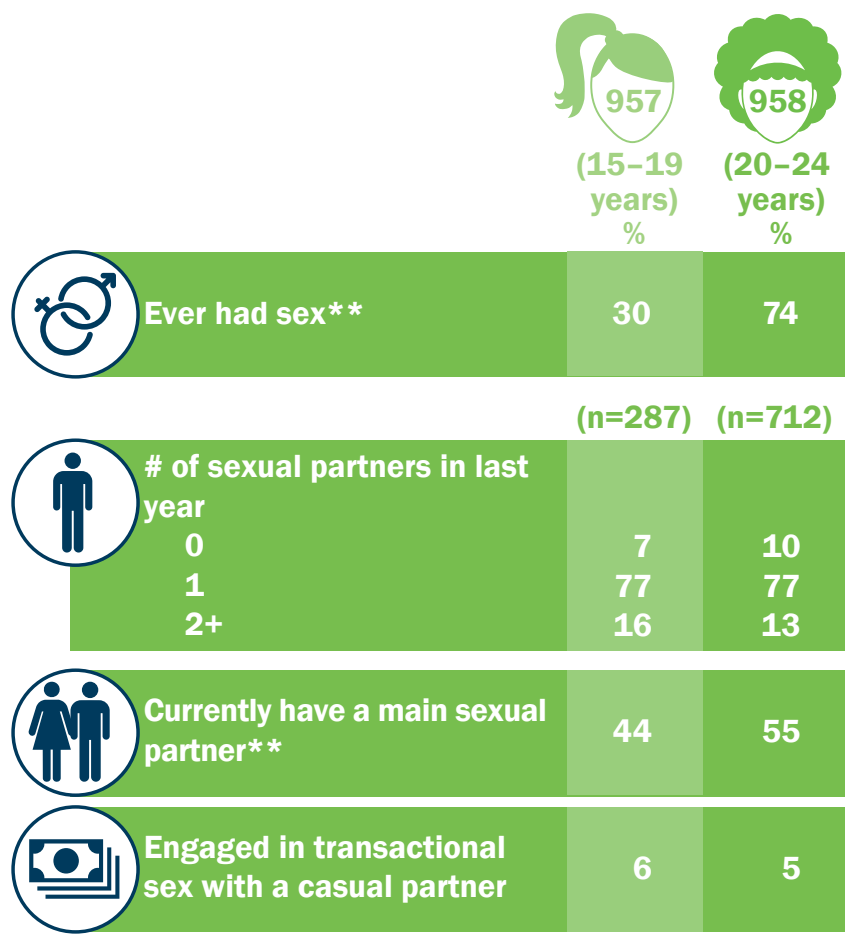

Have AGYW recently tested for HIV, do they know their status, and do they think they are at risk of getting HIV?

Adolescent girls (15-19 years) $(n=957)$

Young women (20-24 years) $(n=958)$

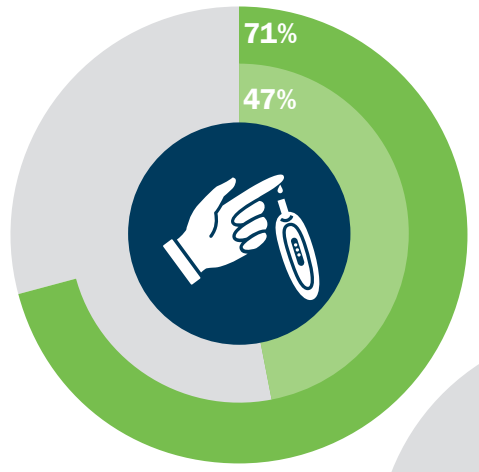

Don't know their HIV status among those who tested in last year ${ }^{* *}$

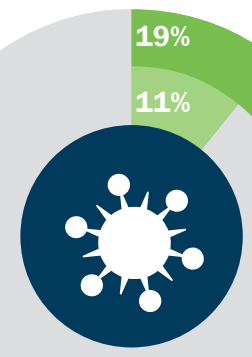

Likely exposed to $\mathrm{HIV}^{* *}$ past year**

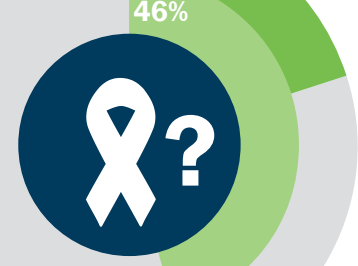

What is the HIV status, STI experience, and service use among AGYW?

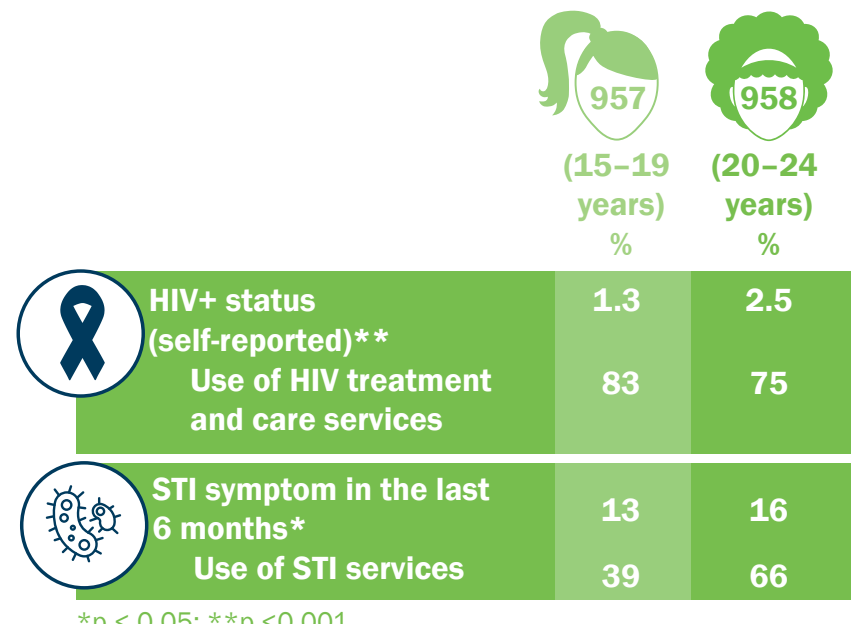

What do AGYW tell us about their male partners?
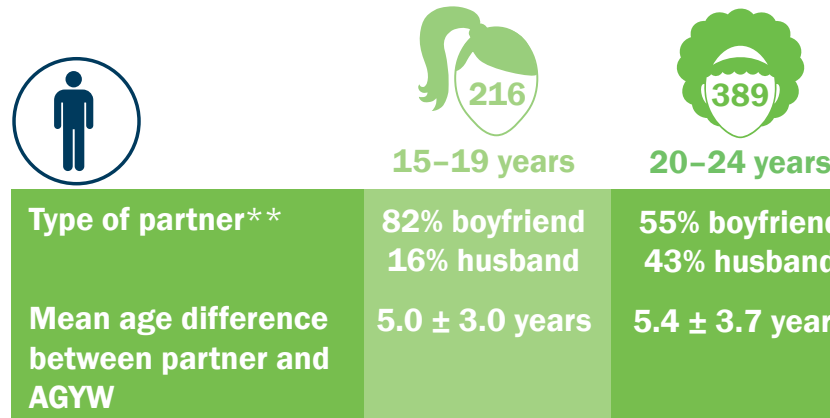

$82 \%$ boyfriend $55 \%$ boyfriend $16 \%$ husband $43 \%$ husband

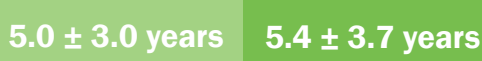

AGYW

Age range

Partner circumcised

Lives in the same

household as AGYW**

Lives in the same

community as AGYW

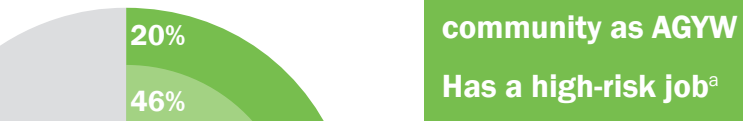

$16-40$ years

19-55 years

${ }^{* *} \mathrm{p}<0.001$

${ }^{a}$ Defined as job categories associated with a greater risk of HIV acquisition and high HIV prevalence (e.g., truckers, laborers). 
What do AGYW tell us about the characteristics of their current relationships?

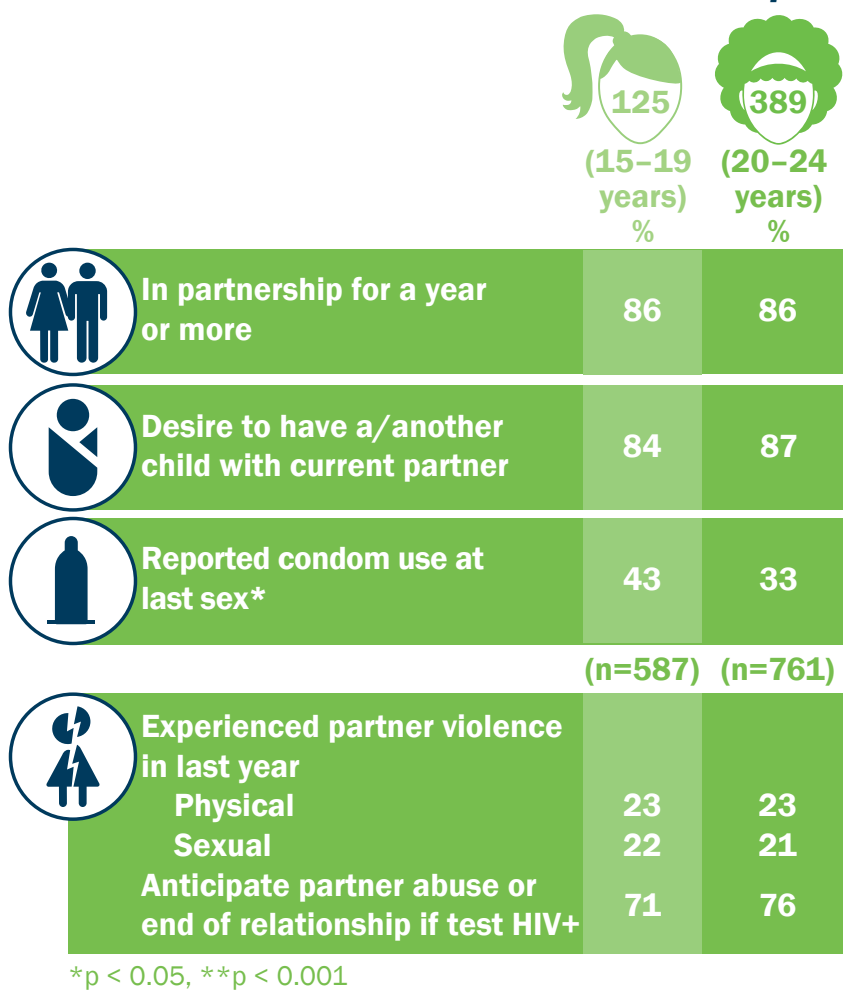

What do AGYW know about partner's HIV risk?

Adolescent girls (15-19 years) $(n=125)$

Young women (20-24 years) $(n=389)$

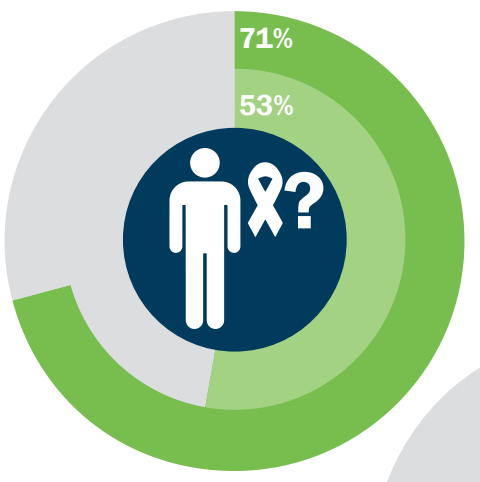

Aware of partner having other partners in the last year

\section{Know their partner's} HIV status ${ }^{\star \star}$

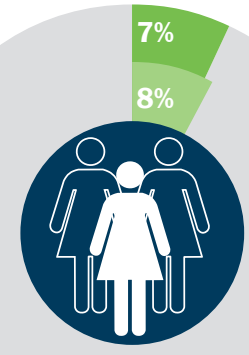

\section{RECOMMENDATIONS}

- Strengthen comprehensive HIV and AIDS education for AGYW that includes building skills to effectively negotiate condom use with their partners.

- Expand strategies to reach AGYW, particularly adolescent girls, with HIV testing and counseling.

The Population Council's It's All One Curriculum is a comprehensive curriculum for program developers that focuses on helping adolescents develop the capacity for healthy, respectful relationships and protect themselves from unwanted and unsafe sex, unintended pregnancy, and STIs.

Available at: popcouncil.org/itsallone

- Strengthen linkages to STI screening and care services for AGYW to reduce risk from potential co-morbidities.

- Assess both partner and relationship characteristics to comprehensively understand AGYW's HIV risk.

- Support AGYW in fulfilling their fertility desires while reducing their HIV risk.

- Invest in strategies to prevent intimate partner violence and mitigate its impacts on AGYW.

- Strengthen HIV counseling and testing for couples, including addressing potential abuse and relationship break-up.
POPULATION COUNCIL

Ideas. Evidence. Impact.
The Population Council confronts critical health and development issues-from stopping the spread of HIV to improving reproductive health and ensuring that young people lead full and productive lives. Through biomedical, social science and public health research in about 50 countries, the Council works with our partners to deliver solutions that lead to more effective policies, programs, and technologies to improve lives worldwide. Established in 1952 and headquartered in New York, the Council is a nongovernmental, nonprofit organization with an international board of trustees. popcouncil.org

Suggested citation: Population Council. 2018. "What do we know about the HIV risk characteristics of adolescent girls and young women in Zambia?: findings from DREAMS implementation science research," DREAMS Results Brief. Washington, DC: Population Council. 